\title{
Identificación de prácticas ambientales sostenibles ejercidas por la Administración Distrital de la ciudad de Bogotá en los procesos de contratación adelantados por parte de la Secretaría de Ambiente en la última década ${ }^{1}$
}

\author{
Identification of sustainable \\ environmental practices exercised \\ by the District Administration of the \\ city of Bogotá in the contracting \\ processes carried out by the \\ Secretary of the Environment in the \\ last decade
}

Beatriz Eugenia Cardona Arteaga*

\section{Resumen}

El presente artículo académico tiene como objetivo principal demostrar si existen o no prácticas sostenibles ambientales en la contratación pública que adelanta la ciudad de Bogotá o si están

1 Artículo de investigación.

* Abogada, especializada en Derecho Administrativo. Magíster en Contratación Pública y Privada de la Universidad Santo Tomás. 
contempladas en un marco legal a través de las actuaciones administrativas que adelante la ciudad, materializadas estas en tres aspectos básicos: uso racional de recursos naturales, la reducción de cargas contaminantes y el desarrollo de iniciativas ambientales enfocadas hacia la sostenibilidad.

El propósito del objetivo se ejecutó a través del trabajo de identificación de correspondencia entre la política pública de crecimiento verde y consumo sostenible y las acciones concretas desarrolladas en el territorio distrital; se efectuó mediante una interpretación de manera reflexiva de diversos actos administrativos, decretos, resoluciones, circulares, contratos, otrosíes a contratos existentes, convocatorias oficiales o en los procesos bajo la modalidad de selección abreviada de menor cuantía, en la Secretaría Distrital de Ambiente, con un enfoque hermenéutico crítico, manteniendo una estrategia de investigación de revisión documental.

Lo anterior se hizo con el fin de resaltar la importancia de adelantar procesos de contratación en el territorio capital con criterios de sostenibilidad ambiental y de la política de crecimiento verde, que conlleven a beneficios ambientales, sociales y económicos, dando paso a la compensación ambiental efectiva y eficiente de los recursos naturales utilizados.

Por último, se plantea demostrar que se puede utilizar al contrato estatal como instrumento que permita el cumplimiento de una práctica de contratación pública sostenible desde el punto de vista ambiental, teniendo en cuenta la importancia de la figura del contrato en sí misma, debido a que puede fungir como un instrumento de control y, a su vez, puede incentivar por este medio la práctica sostenible la adquisición de bienes y servicios de manera responsable y eficiente al hacer uso de los recursos naturales, disminuyendo los impactos ambientales que ya estamos vivenciando. 


\section{Palabras clave:}

Desarrollo sostenible, compra pública, práctica sostenible de contratación pública, crecimiento verde, compensación ambiental.

\section{Abstract}

The main objective of this academic article is to demonstrate whether or not there are sustainable environmental practices in public procurement carried out by the city of Bogotá or if they are contemplated in a legal framework through the administrative actions carried out by the city, materialized in three basic aspects: rational use of natural resources, the reduction of polluting loads and the development of environmental initiatives focused on sustainability.

The purpose of the objective was executed through the work of identifying the correspondence between the public policy of green growth and sustainable consumption and the concrete actions developed in the district territory, executed through a reflective interpretation of various administrative acts, decrees, resolutions, circulars, contracts, otheri to existing contracts, official calls, or in the processes under the modality of abbreviated selection of smaller amounts, in the District Secretary of Environment, with a critical hermeneutical approach maintaining a document review research strategy.

The foregoing, in order to highlight the importance of advancing contracting processes in the capital territory with criteria of environmental sustainability and a green growth policy, which lead to environmental, social and economic benefits, giving way to effective and efficient environmental compensation. of the natural resources used.

Finally, it is proposed to demonstrate that the state contract can be used as an instrument that allows compliance with a sustainable public procurement practice from an environmental point of view, 
taking into account the importance of the figure of the contract itself, because it can serve as a control instrument, and in turn it can incentivize the sustainable practice of acquiring goods and services in a responsible and efficient manner by making use of natural resources, reducing the environmental impacts that we are already experiencing.

\section{Key words:}

Sustainable Development, Public Procurement, Sustainable Public Procurement Practice, Green Growth, Environmental Compensation.

\section{PRIMERA PARTE:}

\section{La importancia de utilizar el contrato} estatal como instrumento que permita adelantar procesos de contratación con criterio sostenible y componentes (Relación beneficios/compensación)

El objetivo del presente acápite es demostrar, desde el punto de vista teórico, la importancia de utilizar el contrato estatal como instrumento para adelantar procesos de contratación con criterio sostenible a través de las cláusulas y componentes que traigan beneficios al entorno ambiental al hacer uso de esos recursos, y, a su vez, que estas mismas exijan la necesaria compensación ambiental para equilibrar el consumo de los recursos naturales, con el fin de seguir evitando el registro de una huella ecológica negativa, acudiendo al principio de precaución y prevención ambiental y a la definición de contratación basada en criterios ambientales (Wilches-Duran, Rafael E. 2013)2

2 "Aquel sistema donde se indica a los actores relacionados con las etapas de la contratación, sea ésta de carácter público o privado, la incorporación de criterios ambientales en estos, por medio de elementos jurídicamente permitidos y ambientalmente acordes, dentro de los diferentes tipos de contratos establecidos en la legislación, sin contrariar los derechos y responsabilidades de estos y sus entes relacionados en el marco de la gestión integral" (p. 391). 
En materia ambiental existe una preocupación por mitigar las consecuencias del consumismo industrial, que ha generado secuelas en el bienestar de los entornos de los diferentes nichos sociales, como el cambio climático, la lluvia acida, la deforestación, la polución, el desabastecimiento del recurso hídrico, las inundaciones, la actividad minera y los movimientos de tierra de manera abrupta, entre otros fenómenos que son muestras de la necesidad de su protección.

Aunado a lo anterior, existe un aprovechamiento inadecuado de los recursos, además del aumento de producción en masa y la escasa racionalización en materia ambiental por parte de la administración estatal, generando impactos negativos en el medio ambiente $y$, por tanto, una insostenibilidad ambiental. Por esto resulta importante mitigar y controlar el uso de los recursos y estimular la conciencia ambiental para evitar la sobre explotación de los recursos naturales evitando al máximo la generación de consecuencias ambientales desfavorables como las mencionadas anteriormente (Conte Grand y D’Elia, 2018).

Esta es una de las razones por las que se ha estudiado la relación entre la población y su aspiración de satisfacer las necesidades de consumo a través de del crecimiento industrial y comercial de los asentamientos poblacionales, generando la utilización de las materias primas de las cuales se desconoce su procedencia, y la medición de hasta dónde va la capacidad de explotación del proveedor y de donde provienen dichas materias, el afán de crecer económicamente buscando elevar los altos niveles de bienestar sin importar el precio o el coste ambiental provocando en la mayoría de los casos la utilización desmedida de los recursos que en no satisface en totalidad la demanda de la población actual. (Lasso-Ruales, 2018).

Ante esta situación, se explora la imposición de las medidas responsables con el medio ambiente, las cuales deben estar en cabeza de las administraciones públicas de cada territorio; en particular, de- 
bido a que este tipo de medidas tienen un alto impacto en el control del manejo de los recursos al interior de sus territorios a través de la expedición de leyes, decretos, ordenanzas, de acuerdo con lo de su competencia en búsqueda de la aminoración de los impactos en las esferas sociales, culturales, económicas y ambientales, siendo esta la materia que preocupa no solo al ámbito público, sino al privado, teniendo en cuenta que todos comparten un mismo espacio.

Esta situación no debe resultar ajena para los asuntos del Derecho. Podría ser de gran ayuda con el fin de solventar de alguna manera la crisis de sostenibilidad ambiental, teniendo en cuenta su función social, que funge como sistema de control a través del direccionamiento y la guía de conductas y la supervisión del funcionamiento de las instituciones sociales para conseguir integrar, cohesionar y equilibrar acciones responsables en el sector político, y así contener, en gran medida, los daños o perjuicios ambientales causados por el desarrollo de proyectos, en pro de un avance para la humanidad, que en la mayoría de los casos se adelanta sin la debida compensación ambiental, la cual debería tener un grado de proporcionalidad al daño o perjuicio causado (Rivera Zea, 2016).

De continuar con las acciones de consumo de recursos naturales, sin implementar las medidas de prevención, corrección, mitigación, recuperación y restauración eficaz que contenga los efectos negativos, la sociedad se encontrará permanentemente expuesta a los efectos ambientales y seguirá sufriendo las consecuencias que estos han venido dejando.

De lo anterior surgió la inquietud de establecer cómo se consumían los recursos naturales en la localidad, desde la esfera de las instituciones públicas y, en particular, desde la alcaldía de Bogotá. Como resultado de este análisis previo emerge la necesidad de identificar si en realidad se encontraban acciones a nivel local, adelantadas por parte de las autoridades institucionales distritales o de quienes toman las deci- 
siones de en las entidades, tendientes a evaluar, diseñar e implementar practicas sostenibles y políticas públicas de protección ambiental que contengan medidas que disminuyan de alguna forma los impactos ambientales existentes, así como prácticas sostenibles en materia ambiental, utilizando el contrato como un instrumento de control.

Como primera medida, se encuentra, desde el punto de vista normativo, que en el ordenamiento jurídico colombiano existe la obligación natural, constitucional y legal del Estado colombiano de proteger los recursos naturales y permitir el goce de los derechos ambientales y colectivos, reconocidos bajo una protección de rango supra nacional a través de los diferentes tratados y acuerdo suscritos por la nación, así como el deber constitucional y legal de proteger el balance de los recursos naturales con enfoque sostenible; al mismo tiempo, el Estado y su institucionalidad debe dar ejemplo al ser el mayor comprador de los diferentes bienes y servicios que permitan cumplir su fin esencial: cumplir con la satisfacción de las necesidades de interés general, generando garantías de la compensación de los recursos naturales utilizados, ejerciendo acciones que conlleven a la conservación de los recursos y la restauración de su equilibrio a través de la observancia normativa y la implementación de las políticas públicas de protección ambiental ya existentes (Ministerio de Ambiente y Desarrollo Sostenible, 2017).

Por tanto la ejecución del desarrollo sostenible supone un conjunto de beneficios, cargas, obligaciones y responsabilidades, compartidas entre los Estados y los ciudadanos, que se materializa a través de diferentes actividades, entre ellas la planificación de la economía, el establecimiento de modelos productivos sostenibles, el uso de herramientas innovadoras y la implementación de políticas públicas encaminadas a promover practicas amigables, al ser el Estado, el mayor comprador de bienes y servicios, y para el presente caso la ciudad de Bogotá, se destaca la importancia de que esta sea, quien adelante procesos de contratación, por parte de su institucionalidad con criterios de sostenibilidad ambiental, 
que conlleven a beneficios ambientales, sociales y económicos con inclusión de criterios sostenibles en la adquisición de bienes y servicios, en beneficio de los habitantes de esta localidad. (De la Rosa y Contreras, 2019).

De otro lado, se debe destacar la utilidad de la figura del contrato en sí misma, debido a que puede fungir como un instrumento de control, seguimiento y monitoreo a la observancia de las normas y políticas públicas que contengan un mandato de protección ambiental si se utiliza de manera adecuada por parte de la administración distrital; además de ser una herramienta útil que permite la vigilancia y el seguimiento al cumplimiento de las medidas de compensación ambiental, adelantando de manera responsable y eficiente el consumo de los recursos naturales existentes en el territorio. Estas son funciones necesarias para contribuir con el usos sostenible y sustentable de los recursos utilizados, buscando implementar el sentido de la corresponsabilidad, que deben tener todos los partícipes del proceso contractual, teniendo en cuenta que es este, el resultado final de todo el proceso de contratación en sí mismo.

Ese deber de sostenibilidad que tienen los gobiernos se debe ver reflejado de manera directa en los procesos de compra pública, a través de los procesos de contratación que requieren las entidades públicas para su funcionamiento y el cumplimiento de sus fines. Si estas entidades se obligaran a comprar bienes y servicios catalogados verdes, exigirían, a su vez, a los proveedores del proceso de contratación a cambiar su forma de producción y distribución, lo cual le daría un impulso significativo a la economía, generando el aporte social y de concientización acerca de la protección al medio ambiente y esto se vería reflejado de manera directa en la población en general (Ochoa Moreno y Moreno Beltrán, 2015).

Así las cosas, la protección del medio ambiente debería ser el principal objetivo para el Estado, y debería considerarse como uno de los 
asuntos que deben ser abordados bajo el estudio de los contratos mercantiles en Colombia, pues, como ya lo reconoce la doctrina nacional,

(...) el conocimiento que hemos adquirido sobre el impacto que la actividad humana produce sobre el medio ambiente hace que la concepción contemporánea del derecho no pueda evadir la consideración de los asuntos relacionados con la ecología (Arrubla, 2009, p. 35).

Tan es así, que si no se tiene en cuenta la huella ecológica negativa, los problemas de desarrollo sostenible ambiental aumentarán y traerán el desabastecimiento de los recursos naturales, cambios climáticos, la deforestación acelerada, y el agotamiento de recursos no renovables, como el agua, el suelo, la flora y la fauna, causado la mayoría de las veces por desinformación en cómo proceder y aprovechar los recursos de manera legal, asimismo, la mala distribución de estos recursos en los diferentes sectores genera impactos sociales, culturales, económicos y ambientales por parte del distrito. El aumento de producción de bienes y servicios en masa vs. la voluntad ambiental de protección, que crece de manera lenta, para compensar ambientalmente los recursos utilizados es otra de las razones que motiva al legislador o al productor normativo para buscar una protección efectiva de los recursos, aunado a un deber de la correcta administración de los recursos por parte de las instituciones estatales; por lo cual, es un problema no solo para los administrados, sino para el mismo Estado (Alcaldía Mayor de Bogotá, D. C., 2018).

Por esta razón, resulta de alta utilidad fijar criterios en el proceso de contratación para el medio ambiente del entorno local y para el desarrollo económico, teniendo en cuenta que al efectuar medidas de protección, por ejemplo, desde la aplicación del principio de planeación interna por parte de las entidades públicas y externa al ofrecer la convocatorias al público, se evitarían impactos ambientales y daños colaterales en las mismas poblaciones a causa de la malversación de los recursos naturales que generan un sostenimiento en los asentamientos poblacionales en 
dichos territorios; asimismo, se fortalecerían los mercados y producciones y se generaría necesariamente el incremento de competitividad en materia de contratación pública, garantizando así mejores precios y calidad sostenible en el producto, prestando un efectivo servicio público y garantizando que los derechos e intereses de los administrados se cumplan, así como los fines esenciales del Estado, tal como lo establece el artículo 2 de la Constitución Política (Amaya, 2016).

Bajo este escenario, algunos países han creado instrumentos voluntarios de protección ambiental que no tienen como fundamento el poder coercitivo ni coactivo de la administración, sino que, por el contrario, encarnan una forma innovadora y revolucionaria de garantizar el orden público ambiental por medio de los deseos y el querer de los ciudadanos, bajo el concepto de poder astuto, porque no representa el criterio tradicional de las fuerza; más que reprimir o contener, busca influenciar el comportamiento de las personas para cumplir con los fines estatales que persiguen las administraciones públicas (Vaquer, 2018).

Haciendo alusión al poder astuto, la administración pública de la ciudad de Bogotá puede hacer uso del contrato estatal y utilizarlo como un instrumento jurídico que sirva para dar cumplimiento a los fines esenciales del Estado en materia ambiental, teniendo en cuenta su carácter versátil y moldeable a las necesidades de la administración pública, que resulta ser la llamada a identificar las necesidades, diseñar los estudios públicos y contratar los bienes y servicios sin perjudicar el patrimonio de la nación. Todo lo anterior se debe efectuar a partir de las opciones que la legislación en materia de contratación estatal consagró, al establecer distintos tipos de contratos que se diseñaron para el fomento de relaciones bilaterales y la satisfacción de las necesidades básicas de la sociedad.

Sin embargo, a partir de la revisión documental que se ha adelantado en la presente investigación, con el fin de dilucidar si se 
han ejecutado practicas sostenibles en la contratación del distrito por parte de sus entidades, se ha determinado que, en principio, de manera general son pocas las normas nacionales que en materia de contratación hacen alusión a la protección del medio ambiente; no obstante, la fuerza vinculante y obligatoria puede hacer que se observen las disposiciones normativas de protección ambiental que, al ser sustentadas en los principios constitucionales, deberían ser imperativas. De tal magnitud es su importancia, ya que motivan al diseño de las políticas públicas con el fin de que se introduzcan conceptos para adelantar prácticas ambientales sostenibles en la contratación; por tanto, la intención de aplicación se debe convertir en una realidad.

Además de la fuerza vinculante del principio constitucional, como se refirió anteriormente, se encuentra un importante fundamento en el artículo setenta y ocho de la Constitución Política para exigir la inclusión de cláusulas que lleven a prácticas sostenibles de contratación. En esta disposición, el mandato resulta ser más preciso al establecer que la ley regulará el control de calidad de bienes y servicios ofrecidos y prestados a la comunidad, así como el establecer la exigencia de brindar la información que debe suministrar al público en su comercialización; del mismo modo permite establecer quienes serán responsables en la producción y en la comercialización de bienes y servicios, debido a que se podrían utilizar bienes y servicios que atenten contra la salud, la seguridad y el adecuado aprovisionamiento a consumidores y usuarios (De la Rosa y Contreras, 2019).

De otro lado, para materializar las prácticas sostenibles, se encuentra una serie de normas que, no por ser escasas, no son suficientes para empezar a promover la protección ambiental, con base en la aplicación de los principios de precaución y prevención ambiental, contemplados en la Ley 99 de 1993. Es así como se encuentra el artículo 2.2.1.1.1.3.1. del Decreto 1082 de 2015, que señala que el restablecimiento o recuperación ambiental es un elemento que se debe considerar en las fases que se adelantan para llegar al cumplimiento 
del objeto contractual; por tanto, la administración pública, para el caso concreto la ciudad de Bogotá, puede, desde el primer momento, expresar a través de la planeación las condiciones para contratar, los criterios a tener en cuenta, las necesidades que pretende suplir y el modo en que cumplirán los objetivos del contrato, respetando las exigencias legales, pues, para algunos casos se deben aportar autorizaciones, licencias o permisos, que son instrumentos necesarios cuando el objeto de la contratación incluya la realización de una obra. Por tanto, la entidad contratante debería contar con los estudios y diseños que permitan establecer la viabilidad de un proyecto, su impacto ambiental, económico, social y ambiental (De la Rosa y Contreras, 2019).

Sin embargo esta cláusula aplicaría para los contratos donde se exigen condiciones como la evaluación del impacto ambiental y las autorizaciones respectivas, debido al uso de algún recurso como suelo, agua, aire, forestal o de fauna; sin embargo, no todos los contratos se rigen por estas exigencias, puesto que dependen del objeto contractual y el impacto que se tenga sobre los bienes jurídicos tutelados por la administración. Por tanto, la norma no sería en estricto sentido suficiente para lograr la protección ambiental que se busca, ni funcionaría como método compensatorio en caso de aprovechamiento de los recursos.

Sin embargo, pese a los esfuerzos normativos aquí referenciados, el criterio de sostenibilidad ambiental no resulta ser determinante para la selección de contratista, por tanto una propuesta académica a esta situación seria que las entidades contratantes entre los requisitos habilitantes tengan presente la experiencia de prácticas y procesos sostenibles de los proponentes para la ejecución de los contratos, al mismo tiempo que la entidad contratante establezca como dentro de sus intereses favorables la protección al medio ambiente, no en cumplimiento de una norma si no a partir de las necesidades a satisfacer y del objeto contractual que se pretende celebrar, y que consulten los programas distritales existentes en gestión ambiental empresarial (Alcaldía Mayor de Bogotá, PREAD, 2020). 
Otro elemento normativo en contratación estatal que permitiría, desde la planeación interna de la administración pública, incrementar el ejercicio de prácticas sostenibles en los procesos de adquisición de bienes y servicios por parte de las entidades se encuentra de manera clara en la disposición estipulada en el artículo 88 de la Ley 1474 de $2011^{3}$, teniendo en cuenta que establece la relación de costo-beneficio al adelantar este tipo de actuaciones, porque implica obtener las mayores ventajas al momento de adquirir los bienes y servicios, por ende, las entidades estatales están facultadas para establecer dentro de los factores de medición, temas relacionados con el impacto ambiental de la oferta en cuestión.

Es aquí donde el análisis del impacto ambiental adquiere también importancia, porque en gran medida en los contratos de obras públicas -volviendo al ejemplo- se han generado inconvenientes al no contar con los estudios, los permisos, las autorizaciones o licencias requeridas para ejecutarlos, lo que denota un desconocimiento del orden jurídico ambiental, y en muchas ocasiones impide la culminación del objeto contractual, por la imposibilidad que se deriva de la fragilidad de los ecosistemas que rodean un proyecto que iría encaminado a

3 La ley dice:

Artículo 88. Factores de selección y procedimientos diferenciales para la adquisición de los bienes y servicios a contratar. Modifíquese el numeral 2 del artículo 5 de la Ley 1150 de 2007 en el siguiente sentido:

"2. La oferta más favorable será aquella que, teniendo en cuenta los factores técnicos y económicos de escogencia y la ponderación precisa y detallada de los mismos contenida en los pliegos de condiciones o sus equivalentes, resulte ser la más ventajosa para la entidad, sin que la favorabilidad la constituyan factores diferentes a los contenidos en dichos documentos. En los contratos de obra pública, el menor plazo ofrecido no será objeto de evaluación. La entidad efectuará las comparaciones del caso mediante el cotejo de los ofrecimientos recibidos y la consulta de precios o condiciones del mercado y los estudios y deducciones de la entidad o de los organismos consultores 0 asesores designados para ello. En los procesos de selección en los que se tenga en cuenta los factores técnicos y económicos, la oferta más ventajosa será la que resulte de aplicar alguna de las siguientes alternativas:

a) La ponderación de los elementos de calidad y precio soportados en puntajes o fórmulas señaladas en el pliego de condiciones; 0

b) La ponderación de los elementos de calidad y precio que representen la mejor relación de costo-beneficio para la entidad (...). (Ley 1474 de 2011) 
la satisfacción de necesidades materializadas en bienes y servicios que se deben prestar a una comunidad, en específico, en el caso concreto la ciudad de Bogotá.

De acuerdo con lo anterior, se plantea que, si las entidades públicas distritales incrementan la demanda de bienes o servicios con criterios ambientales, el mercado se tendría que acoplar a estos nuevos estándares, aumentado su oferta, disminuyendo costos y dejando abierto el mercado para que los particulares se adhieran a este tipo de mecanismos y procedimientos en pro del medio ambiente. La inclusión de criterios ambientales dentro del marco de la Contratación Pública a nivel distrital conllevaría a beneficios primordiales para el medio ambiente y la economía, permitiendo así el cumplimiento responsable de los fines esenciales del Estado (Eco directorio, PREAD.2020).

Con la observación y cumplimiento de estas normas contractuales se daría cumplimiento al claro mandato constitucional que obliga al Estado y a todas las entidades territoriales, en particular a la ciudad de Bogotá, a tomar todas las medidas para proteger los recursos naturales, de tal forma que la aplicación de las políticas públicas de índole nacional, como las aquí señaladas de crecimiento verde, materializada en las acciones compra publica verde ${ }^{4}$, encuentran suficiente sustento

4 Puntualizando en la definición de compra publica sostenible, Félix Valera Ibáñez la define como:

La adquisición de bienes y/o servicios con criterios ambientales realizada por una entidad estatal en virtud de las facultades de contratación; en otras palabras, es una contratación en la cual se han contemplado requisitos ambientales relacionados con una 0 varias etapas del ciclo de vida del bien y/o servicio por comprar; esto se produce desde la extracción de la materia, su fabricación, distribución y uso hasta su disposición. De este modo, el comprador satisface la necesidad de una institución que da origen a la compra, pero no descuida el impacto ambiental que esta ocasionara. (Ibáñez, 2011).

De otro lado, la adopción del concepto de 'compra públicas verdes', o como se conoce en ingles Green Public Procurement (GPP), se hace más notable toda vez que el país establece criterios ambientales dentro de los procesos contractuales de las entidades públicas. Aunque todavía no se haya desarrollado de manera exponencial, resulta plausible proyectar la imposición de estos criterios de manera generalizada y, porque no, obligatoria en cada proceso contractual que se desarrolle en el territorio nacional y local (Bogotá). (PREAD, PROGRAMA DISTRITAL DE COMPRAS VERDES). 
ético, social, económico y normativo para que sea obligatoria su observación al momento de planear un proceso de contratación para las entidades públicas. De otro lado, el expedir normas en pro de la compensación y protección de los recursos ambientales que sean efectivas, eficientes y eficaces para las sociedad, desde la competencia nacional o territorial, resulta ser un aliciente para los problemas ambientales planteados, buscando dar el uso racional de recursos naturales, la reducción de cargas contaminantes y el desarrollo de iniciativas ambientales, enfocadas hacia la sostenibilidad .

De estas medidas responsables adicionales a las planteadas, se materializa la adopción de las políticas públicas a través de la reglamentación e imposición de la normatividad en pro del desarrollo de las actividades económicas sostenibles, como lo es la política de crecimiento verde. Esta surge por la preocupación de la demanda de la población mundial, que crece de manera acelerada, en contraposición a la disponibilidad de recursos naturales, llegando a un punto crítico frente al uso; por esta razón se debe buscar un balance entre el crecimiento económico, la equidad social y la conservación ambiental que permita llegar a un desarrollo sostenible.

La política enfocada con dimensión ambiental fue el resultado del trabajo adelantado por la Misión de Crecimiento Verde, que permitió su formulación, cuyo objetivo es impulsar para el 2030 el aumento de la productividad y la competitividad económica del país, al tiempo que asegura el uso sostenible del capital natural y la inclusión social, de manera compatible con el clima. Esta política fue aprobada por el Consejo Nacional de Política Económica y Social el 10 de julio de 2018 (CONPES 3934), y hace parte de la misión de crecimiento verde en virtud de las recomendaciones precisas de diseñar e implementar políticas macro y sectoriales para cumplir con los objetivos de desarrollo sostenibles (ODS); en particular, el establecido en el numeral 12, "Producción y consumo responsables", el cual resulta aplicable para el interés de la presente investigación, toda 
vez que este consiste en reducir la huella ecológica mediante un cambio en los métodos de consumo y producción de bienes y servicios a través de nuevas modalidades que sean sostenibles y responsables a nivel solo nacional, sino también con aplicación a las localidades, y en particular a la ciudad de Bogotá.

Para lograr el objetivo se fijaron metas, entre ellas se encuentra la número 12.7, que busca implementar prácticas sostenibles de contratación pública, por tanto, es un deber imperativo para la administración distrital adelantar acciones concretas en sus procesos de contratación y cumplir con estas teniendo en cuenta que los ODS fueron aprobados por el CONPES 3918 de 2018 e incorporados en el Plan nacional de Desarrollo 2018-2022, y que, aunado a lo anterior, existe todo un respaldo normativo que tiene en cuenta la dimensión ambiental a través de y las acciones concretas desarrolladas en el territorio distrital, ejecutada mediante diversos actos administrativos, decretos, resoluciones, circulares, contratos, otrosíes a contratos existentes, convocatorias oficiales o en los procesos bajo la modalidad de selección abreviada de menor cuantía, como se verá en la segunda parte del presente trabajo de investigación.

\section{SEGUNDA PARTE:}

Verificación de los procesos normativos distritales, disposiciones, directrices o políticas que permitan cumplir con las prácticas sostenibles de contratación si se pueden materializar en los procedimientos de contratación pública que adelantan (Línea de tiempo de las actuaciones administrativas)

En la Ciudad de Bogotá se han realizado una serie de actuaciones administrativas encaminadas a establecer la materialización de las disposiciones normativas o a incentivar su desarrollo, con el 
fin de implementar las políticas públicas, normas y procedimientos, buscando la defensa y preservación del medio ambiente. Del mismo modo, se ha podido evidenciar un progreso en la consolidación de un esquema de contratación pública sostenible, identificando que la fase precontractual resulta determinante debido a que es aquí donde inicia el proceso de formación de la voluntad de las partes y se estructura el negocio que se pretende celebrar, aplicando el principio de planeación, identificando la necesidad de aplicar un análisis previo sobre las especificaciones, la oportunidad y conveniencia de su ejecución.

Dicho análisis supone: adelantar estudios previos, tanto técnicos como de mercado, con el fin de determinar la prioridad, lineamientos y finalidades de su elaboración; la obtención de permisos y licencias de conformidad con la Ley aplicable al caso concreto, la determinación de los riesgos previsibles y la maduración de proyectos (Expósito Vélez, 2013). Por tanto, Identificar el cuerpo normativo, las actuaciones administrativas, decretos, resoluciones, circulares, contratos, otrosíes a contratos existentes, convocatorias oficiales y demás resulta importante para desarrollar la pregunta de investigación que orienta este trabajo, en donde se indaga si la Administración Distrital de la ciudad de Bogotá adelanta o no acciones normativas de protección ambiental en los contratos públicos celebrados en pro de un desarrollo sostenible ambiental en el periodo de los años 2010 al 2020, caso de revisión concreto Secretaría Distrital de Ambiente.

Entrando en materia, como primer referente normativo, con aplicación al Distrito en pro de la protección ambiental del territorio de la ciudad de Bogotá, se encuentra el Acuerdo 19 de 1996, "Por el cual se adopta el Estatuto general de protección ambiental del Distrito Capital de Santa Fe de Bogotá y se dictan normas básicas necesarias para garantizar la preservación y defensa del patrimonio ecológico, los recursos y el medio ambiente", el cual tuvo como motivación 
propender por el mejoramiento de la calidad del medio ambiente y los recursos naturales, como mecanismo para mejorar la calidad de la vida urbana y rural y satisfacer las necesidades de los actuales y futuros habitantes del Distrito Capital. Este acuerdo inicial puede fungir como un precedente de obediencia (López Medina, 2007).

Para el operador jurídico, que revisa las actuaciones distritales en pro de la protección ambiental con el fin de ejercer practicas sostenibles en materia de contratación, resulta pertinente tener en cuenta este primer hito, ya que a partir de este acuerdo se genera un sustento para desarrollar nuevos acuerdos que se relacionan con el inicial, como con el Acuerdo Distrital 61 de 2002, por el cual se promueve la implementación de talleres permanentes para el aprendizaje de prácticas de reciclaje dentro de una cultura de gestión integral de residuos sólidos; que resulta ser fundamental en el momento de apropiar la practica sostenible en contratación, ya que el mayor consumo de las entidades públicas radica en el uso del papel y materiales de oficina, que hoy en día se centran en el reciclaje, reutilización y reducción de consumo. (Min ambiente, 2019, CPS).

Con posterioridad surge la Política de Producción Sostenible para Bogotá D. C.; es el instrumento de planeación que establece los lineamientos para el desarrollo de programas distritales de producción sostenible, basado en el equilibrio entre las necesidades sociales, económicas y ambientales de la población y la problemática integral del sector productivo de la ciudad. Fue adoptada mediante el Decreto 482 de 2003, con el objetivo de mejorar la calidad de vida de la población, el entorno ambiental y la competitividad empresarial en la ciudad a través de programas de producción sostenibles en los sectores productivos, considerando el sector servicios e institucional del Distrito.

En el año 2007 la Alcaldía Mayor de Bogotá creó la guía verde de contratación como herramienta interna, en cumplimiento con los 
criterios establecidos para el Plan Institucional de Gestión Ambiental (PIGA), de la misma entidad, según lo dispuesto en el Acuerdo 456 de 2008, "Por el cual se reforma el Plan de gestión Ambiental del Distrito Capital y se dictan otras disposiciones", estableció que las entidades que integran el Sistema Ambiental del Distrito Capital (SIAC) son ejecutoras principales del Plan de Gestión Ambiental, conforme a sus atribuciones y funciones misionales. Adicionalmente, que las demás entidades distritales, organizadas por sectores, son ejecutoras complementarias del Plan de Gestión Ambiental, conforme a sus atribuciones y funciones misionales, en la medida en que contribuyan al cumplimiento de los objetivos y estrategias del mismo desde su PIGA.

El referido Decreto 456 de 2008 estableció como corresponsables de la gestión ambiental del Distrito Capital, de acuerdo con sus deberes, derechos, funciones, competencias, actividades, recursos y posibilidades, a todas las personas naturales y jurídicas ubicadas en el territorio del Distrito Capital que, de manera permanente o temporal, con la asesoría y bajo la coordinación de las autoridades ambientales deben proteger los recursos naturales del país, velar por la conservación de un ambiente sano y propiciar el desarrollo sostenible. Presentó como objetivo general de la gestión ambiental del Distrito Capital propender por el uso racional de los recursos y un ambiente saludable, seguro, propicio, diverso, incluyente y participativo en su territorio para las generaciones presentes y futuras, actuando responsablemente con la región y el planeta.

Asimismo, estableció los objetivos específicos de calidad ambiental, de ecoeficiencia y de armonía socio-ambiental, siendo este uno de los mayores avances para implementar la ejecución de las prácticas sostenibles de contratación de manera incipiente, pero con exigencia normativa. Se considera que el avance que deja esta actuación administrativa radica en la creación del programa de consumo sostenible y el de implementación de prácticas sosteni- 
bles $^{5}$, debido a que materializa de manera concreta el objetivo de desarrollo sostenible (ODS); a su vez, se registra la existencia de acciones de protección ambiental materializadas puntualmente en ejecución de prácticas sostenibles de contratación pública adelantadas por parte de la Secretaría de Ambiente Distrital de la ciudad de Bogotá; por tanto, da una respuesta afirmativa a la hipótesis planteada, al menos en este aspecto ambiental de ejecución.

En otra instancia de la presente revisión académica, sobre la línea de tiempo de expedición de actos administrativos, se encuentra el tercer referente fuerte, frente a las actuaciones adelantadas por las entidades administrativas distritales, encontrando un apoyo a las practicas sostenibles en la expedición del Acuerdo 540 de 2013, expedido por el concejo de Bogotá, el cual estableció los lineamientos del programa distrital de compras verdes con el alcance y el propósito de promover en todas las entidades del Distrito y particulares

5 Para una revisión académica más concreta se puede ver en la parte dispositiva de la Resolución 242 de 2014, expedida por la Secretaría Distrital de Ambiente, en el artículo 13, sobre los diferentes programas de gestión ambiental el sentido de adelantar el Programa de Consumo Sostenible.

Este programa deberá definir acciones que promuevan el uso y consumo responsable de materiales; el fortalecimiento de la cadena de suministro que generen valor agregado en la entidad, con la adquisición de un bien, producto o servicio; que minimice los impactos ambientales más significativos desde la extracción de la materia prima, su fabricación, distribución, hasta su disposición final, considerando el ciclo de vida de los productos. Las entidades deberán verificar que los terceros con que se contrate la prestación de un bien o servicio cuenten con los permisos ambientales requeridos para el desarrollo de su actividad.

Asimismo se definió el sentido del Programa de Implementación de Prácticas Sostenibles. Con este programa las entidades podrán desarrollar actividades o proyectos que contengan uno o varios de los siguientes aspectos: adopción de una cultura ambiental positiva, interacción con temas de interés ambiental, articulación con las políticas, planes o lineamientos distritales, regionales y/o nacionales, que presenten características como ser innovadores, con alto potencial de transferencia, que generen valor agregado a la entidad, que sean reconocidas como experiencias exitosas por actores claves o que incluya aspectos de sostenibilidad. Las actividades o proyectos de este programa deben considerar un modelo sostenible que contenga aspectos que lleven a la protección del ambiente, la calidad de vida de los ciudadanos y el desarrollo económico. Como parte de este programa, la entidad deberá calcular la Huella de Carbono Corporativa, de acuerdo con los lineamentos de la Secretaría Distrital de Ambiente. 
que prestan servicios públicos la vinculación de los propósitos de las compras verdes, entendidas como

(...) el proceso mediante el cual las autoridades públicas tratan de adquirir bienes, servicios y obras con un impacto ambiental reducido durante todo su ciclo de vida en comparación con los bienes, servicios y obras con la misma función principal que normalmente se hubiera adquirido (art. 2, Acuerdo 540 de 2013).

En razón a esta disposición, se dio un lugar a la creación del programa distrital de compras verdes para el fortalecimiento Compras Públicas Sostenibles (CPS). De este esfuerzo político, el Ministerio del Medio Ambiente manifestó en la guía conceptual y metodológica de las CPS que se destacaba la asignación de responsabilidad de la formulación del programa a la Secretaría Distrital del Medio Ambiente en coordinación con las entidades que estime pertinentes, además de promover las compras verdes en todas las entidades del Distrito que prestan servicios públicos, especificando los siguiente ámbitos de aplicación: 1. Evaluación de proveedores, a quien le compro; 2. la evaluación de bienes y servicios, qué compro 3; evaluación metodológica de compra, cómo compro. Por esto, el impacto resultó ser el logro de crear un contexto de voluntad política e incorporación de las CPS en documentos estratégicos, fomentando la discusión, desarrollo e inclusión de parámetros referentes a las CPS a nivel local (Ministerio de Ambiente y Desarrollo Sostenible, 2017).

Asimismo, la Secretaria de Ambiente Distrital manifiesta que la Organización para la Cooperación y el Desarrollo Económico (OCDE) ha precisado esfuerzos para la modernización de la institucionalidad en el país, y sugiere que se requerirán ajustes al ordenamiento jurídico colombiano, que involucren aspectos tales como la incorporación de criterios en la contratación pública que garantice la transparencia, la no discriminación y la competencia, así como los impactos ambientales y sociales a lo largo del ciclo de vida y la gene- 
ración de herramientas para la información, capacitación, asistencia técnica, evaluación y mejora de las políticas de compra pública, con el fin de garantizar que sean económicamente eficientes, socialmente justas y ambientalmente eficaces. (oab.ambientebogota, 2020).

Aunado a lo anterior, se encuentra en la página web de la Secretaría de Ambiente Distrital la información pertinente sobre la referida política de compra pública sostenible y su relación con la ejecución de prácticas sostenibles, con el ánimo de promocionar la gestión ambiental en los procesos de contratación, y refieren que desde el año 2014 se ha dado lugar al programa de Consumo Sostenible, el cual define acciones que promueven el uso y consumo responsable de materiales, adquiriendo bienes que minimicen los impactos ambientales más significativos desde la extracción de la materia prima, su fabricación, distribución hasta su disposición final, considerando el ciclo de vida de los productos, requiriendo también que las entidades verifiquen que los terceros con quienes hacen las contrataciones o adquisiciones de un bien o servicio cuenten con los permisos ambientales solicitados para el desarrollo de su actividad donde se asegura el cumplimiento de este programa.

Desde el área de la ejecución y la práctica a través de la materialización de hechos concretos, la Secretaría Distrital de Ambiente, en conjunto con otras entidades, manifiestan haber formulado el Programa Distrital de Compras Verdes, brindando de esta forma las bases para que las entidades desarrollen sus procesos de compras y contratación, enfatizando las Compras Públicas Sostenibles como el proceso mediante el cual se buscan adquirir bienes, servicios y obras con un impacto ambiental reducido durante todo su ciclo de vida, en comparación con los bienes, servicios y obras con la misma función principal que normalmente se hubiera adquirido.

De acuerdo con el reporte informativo en la página web del Observatorio Ambiental de Bogotá, de la Secretaría de Ambiente, 
se muestra como un avance importante de la implementación del Programa Distrital de Compras Verdes, y en general de las Compras Públicas Sostenibles, que las entidades han acogido diversidad de cláusulas ambientales en sus contratos; algunas específicas relacionadas con criterios ambientales puntuales para el bien o servicio a contratar, y otras más generales con relación al cumplimiento de los Planes Institucionales de Gestión Ambiental, por parte de los servidores públicos.

De acuerdo con la información adelantada por la entidad distrital ambiental, se encuentra que, según el reporte, las entidades del orden territorial han desarrollado documentación que permite avanzar en el tema de manera más organizada y formal; dado que esto incluye la generación de fichas técnicas para la adquisición de bienes o servicios específicos con criterios ambientales, la expedición de guías que permiten orientar, básicamente al área contractual y ambiental de la entidad en este proceso, y procedimientos y manuales que especifican los pasos a seguir para hacer las compras de manera amigable con el ambiente ${ }^{6}$.

Otra de las gestiones administrativas encontradas en la presente búsqueda académica está adoptada mediante el Decreto 566 de 2014, en la cual se establece que la Política Pública de Eco Urbanismo y Construcción Sostenible (PPECS) se constituye en un instrumento estructural, mediante el cual se direccionan acciones de orden técnico, institucional, económico y social que generan lineamientos complementarios para los instrumentos de planeación, con la finalidad de reorientar las actuaciones de urbanismo y construcción de Bogotá D. C. hacia un enfoque de desarrollo sostenible, contribu-

6 Este indicador refiere el número de entidades públicas distritales que incluyen cláusulas ambientales en sus procesos de contratación. Los datos empleados para el cálculo del indicador a nivel distrital son reportados por las entidades acogidas al PIGA mediante el informe "Verificación" a través de la herramienta sistematizada STORM de los periodos comprendidos entre enero-junio y julio-diciembre. 
yendo con la transformación de un territorio resiliente, que mitiga y se adapta al cambio climático.

Esta política surge como respuesta al desarrollo insostenible de la ciudad, al reconocer que las dinámicas del crecimiento urbano y los procesos constructivos han contribuido a la segregación socio espacial y al deterioro de los ambientes naturales y construidos, disminuyendo la capacidad de (autorrecuperación) resiliencia del territorio, en detrimento de la calidad de vida de los habitantes urbanos y rurales.

Otra de las figuras necesarias para adelantar practicas sostenibles en los procesos de contratación se encuentra en el Decreto 165 del 11 de mayo de 2015, "Por el cual se reglamenta la figura de Gestor Ambiental para las entidades distritales, prevista en el Acuerdo 333 de 2008, y se dictan otras disposiciones”, estableciendo la figura de Gestor Ambiental en todas las entidades del Distrito Capital, con el objetivo principal de realizar acciones conducentes a la reducción de los costos ambientales, producidos por sus actividades.

Por último, como otra figura de respaldo a la práctica, se encontró que la Secretaría Distrital de Ambiente exige que, para adelantar sus procesos de contratación, se debe incluir dentro de todos los contratos que celebren las dependencias o instituciones distritales una "cláusula ambiental" para la cual se propone el siguiente texto:

El contratista debe cumplir con los lineamientos ambientales de la entidad, descritos en el PIGA; el incumplimiento de esta cláusula por parte del contratista ocasionará terminación unilateral del contrato y se hará efectiva la póliza de calidad del mismo.

El Plan Institucional de Gestión Ambiental (PIGA), como instrumento de planeación, ha sido la herramienta utilizada por el Distrito Capital, a través de su Secretaría de Ambiente, para imple- 
mentar las CPS, incluyendo dentro de él un programa específico de Consumo Sostenible con las referencias, alusiones, compromisos, obligaciones y deberes sobre las CPS que debe observar las instituciones distritales y sus funcionarios.

El Distrito capital a nivel local resulta ser el mayor comprador de bienes y servicios, razón por la cual puede ejercer una posición dominante positiva en el mercado si obliga al cumplimiento y se incluye como clausula obligatoria las condiciones de sostenibilidad ambiental y sus prácticas en el proceso contractual al participante del proceso. Esto se lograría desde el momento en que el distrito proyecte la necesidad del bien o el recurso para el territorio, hasta la inclusión de lo dispuesto en el acuerdo en la elaboración de los estudios previos, y la estructura de cada contrato que se celebre en su territorio, de esta manera se exigiría al proveedor de los insumos necesitados a que participen en los procesos de selección contractuales, utilizando como materia prima productos amigables con el medio ambiente, cambiando su forma de producción, distribución, uso y disposición final, generando así una nueva cultura de consumo.

Por tanto, los criterios de sostenibilidad son una variable o requisito asociado a un aspecto ambiental, social o económico en el ciclo de vida, por medio del cual se desea lograr la desaparición, minimización o control de los efectos adversos en el medio ambiente y la sociedad, en las modalidades de contratación, a saber: licitación pública, selección abreviada, concurso de méritos, contratación directa, mínima cuantía y enajenación de bienes del Estado, que se pueden exigir en cada modalidad. La norma contractual colombiana fija los mecanismos para determinar la oferta más favorable de acuerdo con la modalidad de selección.

Para las modalidades de licitación y selección abreviada de menor cuantía, la entidad puede optar por ponderar elementos de calidad y 
precio soportados en puntajes, fórmulas o ponderar elementos de calidad y precio que representan la mejor relación del costo-beneficio, en cuyo caso los criterios de sostenibilidad cobran gran relevancia, pues son estos los más útiles para fijar tal relación.

En concreto, al definir los criterios de selección, que como se ha dicho son factores calificables, estos podrán ser criterios de sostenibilidad u otros criterios que recaigan siempre sobre la oferta, pudiendo también establecerse todos o algunos como obligatorios y algunos como preferibles.

En cada modalidad y cada proceso de contratación, la ubicación de los criterios de sostenibilidad ambiental podrán variar según la forma en que cada entidad establezca sus necesidades, las condiciones generales del contrato, los requisitos mínimos habilitantes y diseñe sus estudios previos, pliegos de contratación y contrato de manera definitiva; criterios que gozan de alguna manera de la discrecionalidad de la entidad para determinar su forma y presentación con unos contenidos mínimos señalados en la norma.

Para el objeto de la presente investigación, se escoge la modalidad de contratación de selección de Mínima Cuantía adelantada por la Secretaría de Ambiente Distrital (teniendo en cuenta que el ejemplo se espera precisamente en dicha dependencia), dado que en un procedimiento sencillo y rápido para escoger al contratista en la adquisición de los bienes, obras y servicios se puede identificar si en la modalidad de selección, que tiene menos formalidades que las demás, así como características especiales, se aplican los criterios de sostenibilidad ambiental, vigilando por demás las reglas aplicables a la modalidad de Selección de Mínima Cuantía, que son las consagradas en el numeral 5 del artículo 2 de la Ley 1150 de 2007, modificado por el artículo 94 de la Ley 1474 de 2011, y demás normas concordantes. Los resultados evidenciados serán expuestos como parte de las conclusiones de la presente revisión. 
Finalmente, resulta pertinente evidenciar la utilidad de las prácticas sostenibles en el marco de la contratación estatal, en particular de cómo estas influyen de manera positiva en el proceso de ejecución de las compras públicas sostenibles y si estas se encuentran enfocadas o no al cumplimiento de los objetivos la agenda 2030, siendo este el objetivo más actual en la implementación de la política pública de crecimiento verde, adoptado por la nación y el cumplimiento de los ODS; asimismo, verificar qué acciones ha adelantado el gobierno para lograr implementar dichas prácticas y si dichas actuaciones son satisfactorias, si logran imponer un control y si, en efecto, evitan en algún porcentaje un desabastecimiento de los recursos naturales a nivel local y regional, con el fin de compensar ambientalmente el desbalance en el cual nos encontramos inmersos como sociedad expectante.

No obstante lo anterior, resulta pertinente adelantar algún seguimiento a la inclusión de políticas públicas y vigentes, teniendo en cuenta que en el plan distrital de desarrollo para los años 20202024, aprobado por el Concejo de Bogotá, mediante acuerdo 761 de 2020, no se incorporan de manera específica en su articulado los 19 objetivos ambientales, los cuales se agrupan en tres categorías: calidad ambiental, eco eficiencia y armonía socio ambiental, establecidos para la ciudad de Bogotá en el Plan de Gestión ambiental (PGA)7 2008-2038; y las 9 estrategias a saber: investigación; información y comunicaciones; educación ambiental; participación; for-

El Plan de Gestión Ambiental -PGA es el instrumento de planeación ambiental de largo plazo de Bogotá, D.C. en el área de su jurisdicción, que permite y orienta la gestión ambiental de todos los actores estratégicos distritales, con el propósito de que los procesos de desarrollo propendan por la sostenibilidad en el territorio distrital y en la región.

Cualquier estrategia e intervención sobre el territorio debe corresponder y estar conforme con los lineamientos ambientales del PGA, el cual se hace operativo a través de los instrumentos de planeación ambiental de corto plazo y de alcances específicos. Su revisión, cada diez (10) años, será coordinada por la autoridad ambiental. (s.f.) 
talecimiento institucional; sostenibilidad económica; cooperación y coordinación interinstitucional; control y vigilancia; manejo físico y eco urbanismo que orientan la gestión ambiental en el Distrito Capital hacia el logro de los objetivos ambientales, a la vez que perfilan el método particular por el cual opta el Plan de Gestión Ambiental para desarrollarlos, de acuerdo a los instrumentos de planeación ambiental, como el Plan de Acción Cuatrienal Ambiental (PACA), el Plan Ambientales Locales (PAL), el Plan Institucionales de Gestión Ambiental (PIGA) y los operativos de planeación ambiental ${ }^{8}$. La observación de no incorporación la efectuó la contraloría distrital el 18 de mayo de la presente anualidad, a la administración distrital actual.

\section{Conclusiones}

A partir de la revisión documental que se ha adelantado en la presente investigación, con el fin de dilucidar si se han ejecutado practicas sostenibles en la contratación del distrital por parte de sus entidades, se ha determinado que, en principio, de manera general son pocas las normas nacionales que en materia de contratación hacen alusión a la protección del medio ambiente; no obstante, si se le imprime la fuerza vinculante y obligatoria como instrumento de llamado a la conciencia de protección ambiental, puede resultar que se observen las disposiciones normativas de protección ambiental, entendiendo que al estar sustentadas en principios constitucionales revisten un carácter de ser imperativas.

8 http://ambientebogota.gov.co/web/portal-siac/instrumentos-de-planeacion-ambiental; Los Instrumentos operativos de planeación ambiental: Son los instrumentos que operativizan e implementan de manera directa el Plan de Gestión Ambiental, a través de la formulación y seguimiento de planes de acción de corto plazo, cuyos principales responsables son las entidades distritales. Dentro de estos instrumentos se encuentran el Plan de Acción Cuatrienal Ambiental -PACA, Ios Planes Ambientales Locales -PAL y los Planes Institucionales de Gestión Ambiental -PIGA. Se caracterizan por no direccionarse a recursos naturales específicos (como los PMA o los POMCA), sino a escalas político-administrativas en general, como la ciudad, las localidades y las entidades distritales. Además, porque priorizan e incluyen la dimensión ambiental en los periodos de armonización de la Administración Distrital. 
De tal magnitud es su importancia que el espíritu de la norma motiva al operador jurídico y administrativo a diseñar políticas públicas con el fin de que se introduzcan conceptos y disposiciones para adelantar prácticas ambientales sostenibles en la contratación; por tanto, la intención de aplicación se debe materializar a una realidad, la cual puede ser llevada a cabo a través de un ejercicio de conciencia social ambiental.

Se deja como observación a quien encuentre interés por este tema que resulta de gran utilidad fijar criterios sostenibles en la construcción de disposiciones normativas o actuaciones administrativas que regulen el proceso de contratación para el medio ambiente del entorno local y para el desarrollo económico, teniendo en cuenta que al efectuar medidas de protección, por ejemplo, desde la aplicación del principio de planeación interna por parte de las entidades públicas, y externa al ofrecer la convocatorias al público, se evitarían impactos ambientales y daños colaterales en las mismas poblaciones a causa de la malversación de los recursos naturales que generan un sostenimiento en los asentamientos poblacionales que habitan en dichos territorios. Asimismo, se fortalecerían los mercados y producciones y se generaría necesariamente el incremento de competitividad en materia de contratación pública, garantizando así mejores precios y calidad sostenible en el producto, prestando un efectivo servicio público y garantizando que los derechos e intereses de los administrados se cumplan, así como los fines esenciales del Estado, tal como lo establece el artículo 2 de la Constitución Política.

Por último, haciendo alusión al poder astuto, la administración pública de la ciudad de Bogotá podría hacer uso del contrato estatal y utilizarlo como un instrumento jurídico que sirva para dar cumplimiento a los fines esenciales del Estado en materia ambiental, teniendo en cuenta su carácter versátil y moldeable a las necesidades de la administración pública, que resulta ser la llama- 
da a identificar las necesidades, diseñar los estudios públicos y contratar los bienes y servicios sin perjudicar el patrimonio de la nación, haciendo uso de las opciones que la legislación en materia de contratación estatal, consagradas al establecer distintos tipos de contratos diseñados para el fomento de relaciones bilaterales y la satisfacción de las necesidades básicas de la sociedad.

Cuando el criterio de sostenibilidad ambiental no resulta ser determinante para la selección de contratista, una propuesta académica sería que las entidades contratantes entre los requisitos habilitantes tengan presente la experiencia de prácticas y procesos sostenibles de los proponentes para la ejecución de los contratos, al mismo tiempo que la entidad contratante establezca como dentro de sus intereses favorables la protección al medio ambiente, no en cumplimiento de una norma, sino a partir de las necesidades a satisfacer y del objeto contractual que se pretende celebrar, y que consulten los programas distritales existentes en gestión ambiental empresarial.

Es una realidad que el objetivo de adelantar practicas sostenibles ambientales en la contratación pública puede cumplirse. El Estado colombiano fijó unas metas a partir de los ODS; entre ellas se encuentra la numero 12.7, que busca implementar prácticas sostenibles de contratación pública. Por tanto, en esta investigación se llega a la conclusión de que es un deber imperativo para la administración distrital adelantar acciones concretas en sus procesos de contratación y cumplir con estas, teniendo en cuenta que los ODS fueron aprobados por el CONPES 3918 de 2018 e incorporados en el Plan Nacional de Desarrollo 20182022; asimismo se debe tener en cuenta el respaldo normativo de la dimensión ambiental ejecutado mediante diversos actos administrativos, decretos, resoluciones, circulares, contratos, otrosíes a contratos existentes, convocatorias oficiales o en los diferentes procesos de contratación. 


\section{Referencias}

Acuerdo 540 de 2013 (2013). Consejo de Bogotá, D. C. Registro Distrital 5268. https:// www.alcaldiabogota.gov.co/sisjur/normas/Norma1.jsp? $i=56074$

Alcaldía Mayor de Bogotá, D. C. (2018). Bogotá. Ciudad Inteligente. Alcaldía Mayor de Bogotá, D. C. https://bogota.gov.co/sites/default/files/inline-files/doc_smartcity.pdf.

Secretaría Distrital de Ambiente (2013). Programa distrital de compras verdes. Lineamientos para la gestión de las compras sostenibles. Bogotá, D. C.: Secretaría Distrital de Ambiente y Alcaldía Mayor de Bogotá, D. C.

Amaya, C. (2016). El principio de planeación en la contratación estatal, un principio no tipificado. Revista Via luris, (20), 105-119.

Arrubla, J. (2009). Contratos mercantiles, Tomo IV: Contratos contemporáneos. Medellín: Diké.

Bogotá, A. (1995). Bogotá. DC, Sitio Web:Http://www.Ambientebogota.Gov.Co/C/Journal/ View_article_content,

Conte Grand, M., \& D’Elia, V. (2018). Desarrollo sostenible y conceptos “verdes”. Problemas Del Desarrollo, 49(192), 61-84.

De la Rosa, M., \& Contreras, D. (2019) XIX Lecturas sobre Derecho del medio ambiente, el esquema de contratación estatal ambiental y los negocios verdes: herramientas de crecimiento económico y desarrollo sostenible (pp. 463-498). Bogotá: Editorial Universidad Externado de Colombia.

Félix Valera Ibáñez, F. J. (2011). Proyecto de Ley 170.: Gaceta, Senado de la República. Obtenido de: http://www.imprenta.gov.co/gacetap/gaceta.mostrar_documento?p_ tipo $=05 \&$ _ _numero $=170 \& p_{-}$consec $=30941$

Moreno Vásquez, H. (2006). Contratación sostenible. En B. Londoño, G. Rodríguez y G. Herrera (Eds.), Perspectivas del derecho ambiental en Colombia (pp. 390-409). Bogotá: Editorial Universitaria del Rosario.

Lasso-Ruales, A. C. (2018). ¿Qué Es La Contratación Pública Sostenible? Un Estudio de sus inicios, sus avances en Colombia y su concepto (Trabajo de maestría en Derecho Administrativo). Universidad del Rosario, Bogotá, D. C.

Ley 1474 de 2011 (2011). Congreso de la República. Diario Oficial n. ${ }^{\circ}$ 48.128. http://www. secretariasenado.gov.co/senado/basedoc/ley_1474_2011.html 
López Medina, D. (2002). El derecho de los jueces. Bogotá: Legis-Uniandes.

Ministerio de Ambiente y Desarrollo Sostenible (2017). Plan de Acción Nacional de Compras Públicas Sostenibles. https://www.minambiente.gov.co/images/AsuntosambientalesySectorialyUrbana/pdf/compras_p\%C3\%BAblicas/Plan_de_Accion_Nacional de Compras Publicas Sostenibles_2016-2020.pdf

Ochoa Moreno, L. M., \& Moreno Beltrán, I. E. (2015). Análisis de la viabilidad de la implementación de compras verdes en una organización teniendo en cuenta la sustentabilidad en la cadena de suministro (Trabajo de grado en Administración de Negocios Internacionales). Universidad del Rosario, Bogotá, D. C.

Rivera Zea, N. M. (2016). La participación ciudadana como herramienta de sostenibilidad social en la planificación de infraestructuras, estudio de caso en la ciudad de Bogotá (Colombia) (Trabajo de grado de maestría en Planificación y gestión en Ingeniería Civil). Universidad Politécnica de Valencia, Valencia.

Vaquer, M. (2018). El poder administrativo. En M. Vaquer (coord.), Á. Moreno (coord.), A. Descalzo (coord.) y L. Parejo (hom.), Estudios de Derecho Público en homenaje a Luciano Parejo Alfonso, Vol. II (pp. 1243-1271). Bogotá: Tirant lo Blanch.

Wilches Durán, R. E. (2014). La protección del ambiente en el contrato a la luz de las teorías del derecho de contratos. estudio a partir del derecho comercial colombiano. Opinión Jurídica, 13(25), 87-104.

Wilches-Durán, R. E. (2011). Principio ambiental de precaución y contratación mercantil en derecho colombiano. planteamiento del problema. Universitas, (123), 283-313.

http://ambientebogota.gov.co/web/portal-siac/instrumentos-de-planeacion-ambiental ; Los Instrumentos operativos de planeación ambiental.

Observatorio Ambiental de Bogotá (2020) (oab.ambientebogota, 2020). 